\title{
System Theoretic Properties of Platoon-Type Systems
}

\author{
Ruth Curtain, Orest Iftime and Hans Zwart
}

\begin{abstract}
This paper presents readily checkable criteria for several system theoretic properties (stability, approximate and exact controllability, exponential stabilizability) for a particular class of infinite-dimensional systems, the platoon-type systems. These systems are used for modeling infinite platoons of vehicles which have spatially invariant dynamics. Several examples are presented to illustrate the theory. Key words: Spatially distributed systems, infinite-dimensional systems, platoons.
\end{abstract}

\section{INTRODUCTION}

In Freedman et al [8] a stability theory was developed for a very general class of systems defined on a locally compact abelian group $\mathbb{G}$ and taking values in a separable Hilbert space. It relied on a generalization of the known transform theory in Loomis [15] and Rudin [19] to Hilbert space valued functions on $\mathbb{G}$ in [7]. Three decades later, motivated by technological progress in microelectromechanical systems (MEMS) and possible applications to platoons of vehicles, among others, Bamieh et al [2] reconsidered this idea under the name of spatially invariant systems. They considered the class of continuous-time state-space linear systems in the general form

$$
\begin{aligned}
\frac{\partial}{\partial t} z(x, t) & =(A z)(x, t)+(B u)(x, t) \\
y(x, t) & =(C z)(x, t)+(D u)(x, t), \quad t \geq 0,
\end{aligned}
$$

where the spatial co-ordinate $x \in \mathbb{G}$, a locally compact abelian group, for example $\mathbb{G}=\mathbb{Z}, \mathbb{R}, \partial \mathbb{D}, \mathbb{Z}(\bmod n)$ or direct products of finitely many of the latter.

Denote the space of vector-valued functions that are square-integrable with respect to the Haar measure $\mu$ for $\mathbb{G}$ by

$$
\mathfrak{L}^{n}(\mathbb{G}):=\left\{f:\left.\mathbb{G} \rightarrow \mathbb{C}^{n}\left|\|f\|_{2}^{2}=\int_{\mathbb{G}}\right| f(x)\right|^{2} \mathrm{~d} \mu(x)<\infty\right\} .
$$

For example, for $\mathbb{G}=\mathbb{R}$ we have $\mathfrak{L}^{n}(\mathbb{G})=\mathbf{L}_{2}\left(0, \infty ; \mathbb{C}^{n}\right)$ and for $\mathbb{G}=\mathbb{Z}$ we have $\mathfrak{L}^{n}(\mathbb{G})=\ell_{2}\left(\mathbb{C}^{n}\right)$ and for $\mathbb{G}=\partial \mathbb{D}$ (the unit circle) we have $\mathfrak{L}^{n}(\mathbb{G})=\mathbf{L}_{2}\left(\partial \mathbb{D} ; \mathbb{C}^{n}\right)$. The state, input and output spaces are $Z=\mathfrak{L}^{n}(\mathbb{G}), U=\mathfrak{L}^{m}(\mathbb{G}), Y=\mathfrak{L}^{p}(\mathbb{G})$, respectively and the operators $A, B, C, D$ are linear, possibly unbounded, operators. Moreover they must all be spatially invariant.

Denote by $T_{a}$ the spatial translation by $a \in \mathbb{G}$ given by

$$
\left(T_{a} f\right)(x)=f(x-a) .
$$

Ruth Curtain is with the Department of Mathematics, University of Groningen, P.O. Box 800, 9700 AV, Groningen, The Netherlands R.F.Curtainerug.nl

Orest Iftime is with the Department of Economics and Econometrics, University of Groningen, Landleven 5, 9747 AD, Groningen, The Netherlands $O$.v. iftimeerug.nl

Hans Zwart is with the Department of Applied Mathematics, University of Twente, P.O. Box 217, 7500 AE, Enschede, The Netherlands h.j.zwartemath. utwente.nl
Then we say that $A$ is spatially invariant if for all $a \in \mathbb{G}$

$$
T_{a}: D(A) \rightarrow D(A) \text { and } A T_{a} f=T_{a} A f \quad \forall f \in D(A) .
$$

The idea is that by applying the Fourier transform to (1) one can obtain a simpler system defined on the character group $\hat{\mathbb{G}}$ of $\mathbb{G}$.

$$
\begin{aligned}
\frac{\partial}{\partial t} \check{z}(\gamma, t) & =(\check{A} z)(\gamma, t)+(\check{B} u)(\gamma, t) \\
& =\check{A}(\gamma) \check{z}(\gamma, t)+\check{B}(\gamma) \check{u}(\gamma, t) \\
\check{y}(\gamma, t) & =(\check{C} z)(\gamma, t)+(\check{D} u)(\gamma, t) \\
& =\check{C}(\gamma) \check{z}(\gamma, t)+\check{D}(\gamma) \check{u}(\gamma, t), \quad t \geq 0, \quad \gamma \in \hat{\mathbb{G}},
\end{aligned}
$$

where $\breve{A}(\gamma), \breve{B}(\gamma), \check{C}(\gamma)$ and $\check{D}(\gamma)$ are multiplicative operators. In our case they are all bounded operators.

In order to analyze and solve problems related to systems of this type, it is natural to apply the theory for infinite-dimensional systems from [4], [5]. In this paper we do this analysis for the special case of the infinitedimensional platoon-type systems for which $\mathbb{G}=\mathbb{Z}$ (with the character group $\hat{G}=\partial \mathbb{D}$ ). We are interested in this type of systems because many related problems are far from being completely solved. We develop new readily testable criteria for system theoretic properties as stability, exact and approximate controllability and observability, stabilizability and detectability. These results are illustrated by several examples.

In Section II we describe the infinite platoon-type systems and argue that one can apply the theory from [5] to this particular class. Conditions for exponential and strong stability are presented in Section III. Necessary and sufficient conditions for approximate observability, approximate controllability, exact controllability, exact observability, exponential stabilizability and detectability are derived in Sections IV and V. The conclusions and future research are discussed in Section VI. We recall the definitions and introduce the notations for various frequency-domain spaces in an appendix.

\section{Platoon-TYPE SYSTEMS}

The motivation for studying this special class of system stems from the interest shown in the literature for controlling infinite platoons of vehicles over the years (see [14], [16], [17], [3], [12]). The models obtained for these configurations have the spatially invariant form

$$
\begin{aligned}
& \dot{z}_{r}=\sum_{l=-\infty}^{\infty} A_{l} z_{r-l}+\sum_{l=-\infty}^{\infty} B_{l} u_{r-l}, \quad-\infty \leq r \leq \infty \\
& y_{r}=\sum_{l=-\infty}^{\infty} C_{l} z_{r-l}+\sum_{l=-\infty}^{\infty} D_{l} u_{r-l}, \quad-\infty \leq r \leq \infty,
\end{aligned}
$$


where the vectors $z_{r} \in \mathbb{C}^{n}, u_{r} \in \mathbb{C}^{m}, y_{r} \in \mathbb{C}^{p}$ and the matrices $A_{l} \in \mathbb{C}^{n \times n}, B_{l} \in \mathbb{C}^{n \times m}, C_{l} \in \mathbb{C}^{p \times n}, D_{l} \in \mathbb{C}^{p \times m}$. Using the terminolgy and formalism of [5] we can formulate (3), (4) as a standard state linear system $\Sigma(A, B, C, D)$ with the state space $Z=\ell_{2}\left(\mathbb{C}^{n}\right)$, the input space $U=\ell_{2}\left(\mathbb{C}^{m}\right)$ and the output space $Y=\ell_{2}\left(\mathbb{C}^{p}\right)$ (see the appendix). Note that $Z, U, Y$ are all infinite dimensional and so $z=\left(z_{r}\right)_{r=-\infty}^{\infty} \in \ell_{2}\left(\mathbb{C}^{n}\right)$, $u=\left(u_{r}\right)_{r=-\infty}^{\infty} \in \ell_{2}\left(\mathbb{C}^{m}\right), y=\left(y_{r}\right)_{r=-\infty}^{\infty} \in \ell_{2}\left(\mathbb{C}^{p}\right)$ and $A, B, C, D$ are convolution operators $T$ given by

$$
(T z)_{r}=\sum_{l=-\infty}^{\infty} T_{l} z_{r-l}=\sum_{l=-\infty}^{\infty} T_{r-l} z_{l} .
$$

The product of two operators is also a convolution operation, for example,

$$
(C A)_{r}=\sum_{l=-\infty}^{\infty} C_{l} A_{r-l}=\sum_{l=-\infty}^{\infty} C_{r-l} A_{l},
$$

provided, of course, that this is well-defined. To derive conditions for these operators to be bounded we take Fourier transforms of the typical operator equation (5) (see Definition 7.4).

$$
\begin{aligned}
(\check{T} z)\left(e^{j \theta}\right) & =\sum_{r=-\infty}^{\infty} \sum_{l=-\infty}^{\infty} T_{l} z_{r-l} e^{-j r \theta} \\
& =\sum_{l=-\infty}^{\infty} T_{l} e^{-j l \theta} \sum_{r=-\infty}^{\infty} z_{r-l} e^{-j(r-l) \theta} \\
& :=\check{T}\left(e^{j \theta}\right) \sum_{r=-\infty}^{\infty} z_{r} e^{-j r \theta}=\check{T}\left(e^{j \theta}\right) \check{z}\left(e^{j \theta}\right),
\end{aligned}
$$

where $\check{z}$ is the Fourier transform of $z$ and

$$
\check{T}\left(e^{j \theta}\right):=\sum_{l=-\infty}^{\infty} T_{l} e^{-j l \theta} .
$$

According to Property 7.2 this will define a bounded operator from $\mathbf{L}_{2}\left(\partial \mathbb{D} ; \mathbb{C}^{m}\right)$ to $\mathbf{L}_{2}\left(\partial \mathbb{D} ; \mathbb{C}^{p}\right)$ provided that $\check{T} \in$ $\mathbf{L}_{\infty}\left(\partial \mathbb{D} ; \mathbb{C}^{p \times m}\right)$, i.e., provided that ess sup $\left|\check{T}\left(e^{j \theta}\right)\right|<\infty$ (see Definition 7.1). In this case we denote

$$
\|\check{T}\|_{\infty}:=\underset{0<\theta \leq 2 \pi}{\operatorname{ess} \sup }\left|\check{T}\left(e^{j \theta}\right)\right|<\infty .
$$

Note that the Fourier transform of the convolution product $\check{C A}=\check{C} \check{A}$ is just matrix multiplication.

In our applications we shall often assume the stronger condition $\check{T} \in \ell_{1}\left(\mathbb{C}^{p \times m}\right)$, i.e.,

$$
\|T\|_{1}=\|\check{T}\|_{1}=\sum_{l=-\infty}^{\infty}\left|T_{l}\right|<\infty,
$$

where $|\cdot|$ denotes the matrix spectral norm. In the case that $p=m, \ell_{1}\left(\mathbb{C}^{m \times m}\right)$ is a Banach subalgebra of $\mathcal{L}\left(\ell_{2}\left(\mathbb{C}^{m}\right)\right)$ with convolution as the product operation. The Fourier transformed operators form a subspace of $\mathbf{L}_{\infty}\left(\partial \mathbb{D} ; \mathbb{C}^{n \times m}\right)$ with the norm $\|\cdot\|_{1}$.

Definition 2.1: The operator $\check{T} \in \mathbf{L}_{\infty}\left(\partial \mathbb{D} ; \mathbb{C}^{n \times m}\right)$ is called summable if it has the well-defined expansion $\breve{T}\left(e^{j \theta}\right)=$ $\sum_{l=-\infty}^{\infty} T_{l} e^{j l \theta}$ and $\|\check{T}\|_{1}<\infty$. We denote the space of operators with this property by $\mathbf{L}_{s}\left(\partial \mathbb{D} ; \mathbb{C}^{n \times m}\right)$.
$\mathbf{L}_{s}(\partial \mathbb{D})$ is also known as the Wiener class. It is readily seen that $\ell_{1}\left(\mathbb{C}^{n \times m}\right)$ is isometrically isomorphic to $\mathbf{L}_{s}\left(\partial \mathbb{D} ; \mathbb{C}^{n \times m}\right)$. So they are Banach spaces under the norm $\|\cdot\|_{1}$. Clearly, $\|\check{T}\|_{1} \geq\|\check{T}\|_{\infty}$, and $\check{T}\left(e^{j \theta}\right)$ and is continuous in $\theta$ on $[0,2 \pi]$. In the case that $n=m$ we have that $\mathbf{L}_{s}\left(\partial \mathbb{D} ; \mathbb{C}^{n \times n}\right)$ is a Banach algebra. However, in the subsequent theory we need to work with bounded operators from the Hilbert space $\mathbf{L}_{2}\left(\partial \mathbb{D} ; \mathbb{C}^{m}\right)$ to the Hilbert space $\mathbf{L}_{2}\left(\partial \mathbb{D} ; \mathbb{C}^{p}\right)$, i.e., $\check{T} \in \mathbf{L}_{\infty}\left(\partial \mathbb{D} ; \mathbb{C}^{p \times m}\right)$ and these operators are not in the Wiener class in general. Moreover, they need not be continuous or defined for all $\theta \in[0,2 \pi]$.

Now, $\ell_{2}\left(\mathbb{C}^{m}\right)$ is isometrically isomorphic to $\mathbf{L}_{2}\left(\partial \mathbb{D} ; \mathbb{C}^{m}\right)$ under the Fourier transform which we denote in this case by $\mathfrak{F}$ (notation used only here for the simplicity of writing its inverse). Hence

$$
\check{u}=\mathfrak{F} u, u=\mathfrak{F}^{-1} \check{u}, \mathfrak{F}(T u)=\mathfrak{F} T \mathfrak{F}^{-1} \mathfrak{F} u, T=\mathfrak{F}^{-1} \check{T} \mathfrak{F},
$$

and $T$ is bounded if and only if $\breve{T}$ is. They have the same norms, since

$$
\begin{aligned}
\|T\| & =\sup _{\|u\|_{\ell_{2}=1}}\|T u\|_{\ell_{2}}=\sup _{\|u\|_{\ell_{2}}=1}\left\|\mathfrak{F}^{-1} \check{T} \breve{u}\right\|_{\ell_{2}} \\
& =\sup _{\|\check{u}\|_{\mathbf{L}_{2}(\partial \mathbb{D})}=1}\|\check{T} \breve{u}\|_{\mathbf{L}_{2}(\partial \mathbb{D})}=\|\check{T}\|,
\end{aligned}
$$

where we have used the fact that $\|u\|_{\ell_{2}}=\|\check{u}\|_{\mathbf{L}_{2}(\partial \mathbb{D})}$.

Taking Fourier transforms of the system equations

$$
\begin{aligned}
& \dot{z}(t)=A z(t)+B u(t), \\
& y(t)=C z(t)+D u(t),
\end{aligned}
$$

we obtain

$$
\begin{aligned}
\mathfrak{F} \dot{z}(t) & =\mathfrak{F} A \mathfrak{F}^{-1} \mathfrak{F} z(t)+\mathfrak{F} B \mathfrak{F}^{-1} \mathfrak{F} u(t), \\
\mathfrak{F} y(t) & =\mathfrak{F} C \mathfrak{F}^{-1} \mathfrak{F} z(t)+\mathfrak{F} D \mathfrak{F}^{-1} \mathfrak{F} u(t) .
\end{aligned}
$$

Hence the state linear system $\Sigma(A, B, C, D)$ is isometrically isomorphic to the state linear system $\Sigma\left(\mathfrak{F} A \mathfrak{F}^{-1}, \mathfrak{F} B \mathfrak{F}^{-1}, \mathfrak{F} C \mathfrak{F}^{-1}, \mathfrak{F} D \mathfrak{F}^{-1}\right)=\Sigma(\check{A}, \check{B}, \check{C}, \check{D})$ on the state space $\mathbf{L}_{2}\left(\partial \mathbb{D} ; \mathbb{C}^{n}\right)$ with input and output spaces $\mathbf{L}_{2}\left(\partial \mathbb{D} ; \mathbb{C}^{m}\right)$ and $\mathbf{L}_{2}\left(\partial \mathbb{D} ; \mathbb{C}^{p}\right)$ respectively. Their system theoretic properties are identical (see [5, Exercise 2.5]) and so it suffices to apply the standard theory from [5] to the Fourier transformed system $\Sigma(\check{A}, \breve{B}, \check{C}, \breve{D})$. Contrary to what is suggested in [2], it is not necesssary to develop new first principle proofs. One only needs to apply the standard theory from [5] to this particular example class. In the subsequent sections we do this and arrive at simple tests for system theoretic properties such as stability, stabilizability, controllability and illustrate these with simple examples.

\section{STABILITY PROPERTIES}

To examine the stability properties of a convolution operator given by (5) it is easier to consider its Fourier transform. So, instead of $A$ we consider $\check{A}$. Since $\check{A}$ is a bounded operator, the semigroup $e^{\check{A} t}$ satisfies the spectrum determined growth assumption (see [4, p.74]), i.e.,

$$
\sup \{\operatorname{Re}(\lambda), \lambda \in \sigma(\check{A})\}=\lim _{t \rightarrow \infty} \frac{\log \left\|e^{\check{A} t}\right\|}{t}=\omega_{0} .
$$


Thus $e^{\check{A} t}$ is exponentially stable if and only if

$$
\sup \{\operatorname{Re}(\lambda) \mid \lambda \in \sigma(\check{A})\}<0 .
$$

Conditions for the spectrum of $\check{A}$ are given in Lemma 7.3. In particular, if $\check{A}\left(e^{j \theta}\right)$ is continuous, $\lambda \in \sigma(\breve{A})$ if and only if $\operatorname{det}\left(\lambda I-\check{A}\left(e^{j \theta}\right)\right)=0$ for some value of $\theta$. More precisely

$$
\sigma(\check{A})=\bigcup_{\theta \in[0,2 \pi]} \sigma\left(\check{A}\left(e^{j \theta}\right)\right) .
$$

Lemma 3.1: If $\check{A}\left(e^{j \theta}\right)$ is continuous in $\theta$ on $[0,2 \pi]$, then a necessary and sufficient condition for exponential stability is

$$
\sup \left\{\operatorname{Re}(\lambda) \mid \exists \theta \in[0,2 \pi] \text { s.t. } \operatorname{det}\left(\lambda I-\check{A}\left(e^{j \theta}\right)\right)=0\right\}<0 .
$$

A weaker form of stability is strong stability.

Definition 3.2: A semigroup $T(t)$ is strongly stable if $\lim _{t \rightarrow \infty}\|T(t) z\|=0$ for all $z \in Z$.

In the case that $\check{A}\left(e^{j \theta}\right)=A_{0}$, a constant matrix, we have $\sigma(\check{A})=\sigma\left(A_{0}\right)$ which comprises eigenvalues. However, in general, $\sigma(\breve{A})$ comprises the essential spectrum. If $\breve{A}$ is a scalar function, the essential spectrum of $\check{A}$ is $\{\check{A}(\phi) \mid \phi \in \partial \mathbb{D}\}$ ([9, Corollary 4.4., p.577]). The spectrum of $\check{A}$ can be very complicated, as the following examples show. Moreover, the system can be exponentially stable or only strongly stable.

Example 3.3: Consider the system (3) with $A_{0}=a, A_{1}=c$ and $A_{-1}=b$ and all other $A_{l}=0$ and all $B_{l}=0$.

$\check{A}\left(e^{j \theta}\right)=c e^{-j \theta}+a+b e^{j \theta}=(b+c) \cos \theta+a+j(b-c) \sin \theta$,

for positive constants $b, c$. If $b=c$, we have $\sigma(A)=\sigma(\check{A})=$ $[a-2 b, a+2 b]$. If $b \neq c$, we have $\sigma(A)=\sigma(\check{A})=\left\{c e^{-j \theta}+\right.$ $\left.a+b e^{j \theta} \mid \theta \in[0,2 \pi]\right\}$ (Figure 1, Left). In both cases the spectrum comprises continuous spectrum. If $a+|b+c|<0$ the semigroup $e^{\check{A} t}$ is exponentially stable. If $a=-|b+c|$ the semigroup $e^{A t}$ is not exponentially stable, since $0 \in \sigma(\breve{A})$. However, it is strongly stable. That follows from [1] using the fact that the intersection of $\sigma(\breve{A})$ with the imaginary axis contains only the origin and the uniform boundness with respect to $t$ of the semigroup

$$
\left|e^{\check{A} t}\right|=e^{(b+c) \cos (\theta) t} e^{-|b+c| t} \leq 1 .
$$

Example 3.4: Consider the system (3) with $A_{0}=-5.5$, $A_{1}=1.5, A_{-1}=0.34, A_{-2}=-.46$ and $A_{-3}=-3$ and all other $A_{l}=0$ and all $B_{l}=0$. The spectrum of $\check{A}$ is $\sigma(\check{A})=$ $\check{A}\left(e^{j \theta}\right)$ provided in Figure 1 (Right). The exponential stability of the semigroup follows from the inequality

$$
\left|e^{\check{A} t}\right|<e^{-0.1 t} .
$$

Example 3.5: Consider the system (3) with $A_{0}=$ $\left[\begin{array}{cc}0 & 1 \\ -\beta & -\mu\end{array}\right], A_{1}=\left[\begin{array}{cc}0 & -1 \\ 0 & 0\end{array}\right]$ and all $B_{l}=0$, where $\beta$ and $\mu$ are positive numbers. The transformed system has

$$
\check{A}\left(e^{j \theta}\right)=\left[\begin{array}{cc}
0 & 1-e^{-j \theta} \\
-\beta & -\mu
\end{array}\right] .
$$

and the spectrum is

$$
\sigma(\check{A})=\left\{\frac{1}{2}(-\mu \pm(x(\theta)+j y(\theta))), \theta \in[0,2 \pi]\right\},
$$
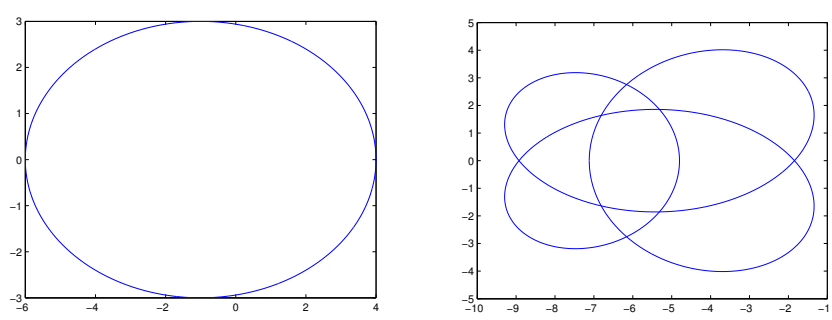

Fig. 1. Left: Spectrum of $\check{A}$ for Example $3.3 b=4, c=1, a=-1$

Right: Spectrum of $\check{A}$ for example 3.4

where $x(\theta), y(\theta)$ are the positive square roots of

$$
\begin{aligned}
2 x(\theta)^{2} & =\sqrt{\left(8 \beta \sin ^{2} \theta / 2-\mu^{2}\right)^{2}+16 \beta^{2} \sin ^{2} \theta} \\
& +\mu^{2}-8 \beta \sin ^{2} \theta / 2 \\
2 y(\theta)^{2} & =\sqrt{\left(8 \beta \sin ^{2} \theta / 2-\mu^{2}\right)^{2}+16 \beta^{2} \sin ^{2} \theta} \\
& -\mu^{2}+8 \beta \sin ^{2} \theta / 2
\end{aligned}
$$

Note that for $\theta=0 x(0)=\mu$ and so $0 \in \sigma(\check{A})$, which shows that $e^{\check{A} t}$ does not generate an exponentially stable semigroup. Since $x(\theta)>0$ for every $\theta \in[0,2 \pi], \check{A}\left(e^{j \theta}\right)$ has two distinct eigenvalues $\lambda_{1,2}(\theta)=\frac{1}{2}(-\mu \pm(x(\theta)+j y(\theta)))$. Then

$$
\check{A}\left(e^{j \theta}\right)=L^{-1}\left[\begin{array}{cc}
\lambda_{1}(\theta) & 0 \\
0 & \lambda_{2}(\theta)
\end{array}\right] L,
$$

where $L$ and its inverse are bounded. Then

$$
\left\|e^{\check{A}\left(e^{j \theta}\right) t}\right\| \leq\|L\|\left\|L^{-1}\right\|\left\|\left[\begin{array}{cc}
e^{\lambda_{1}(\theta) t} & 0 \\
0 & e^{\lambda_{2}(\theta) t}
\end{array}\right]\right\|
$$

If $\mu^{2}-2 \beta>0$ then the inequality $x(\theta) \leq \mu$ is satisfied for all $\theta \in[0,2 \pi]$, which implies that $e^{\lambda_{1,2}(\theta) t}$ are uniformly bounded. Then $e^{\check{A} t}$ is uniformly bounded (with respect to t). The intersection of $\sigma(\check{A})$ with the imaginary axis contains only the origin. From [1], it follows that the semigroup $e^{\check{A} t}$ is strongly stable provided that $\mu^{2}>2 \beta$ and $\beta>0$.

Example 3.6: Consider the system (3) with $A_{0}=$ $\left[\begin{array}{cc}-1 & 1 \\ 0 & -1\end{array}\right], A_{1}=\left[\begin{array}{ll}1 & 0 \\ 0 & 1\end{array}\right]$ and all $B_{l}=0$. The transformed system has

$$
\check{A}\left(e^{j \theta}\right)=\left[\begin{array}{cc}
-1+e^{-j \theta} & 1 \\
0 & -1+e^{-j \theta}
\end{array}\right] .
$$

The system is not exponentially stable because $0 \in \sigma(\check{A})$. Moreover, it is not strongly stable because $\left\|e^{\breve{A} t}\right\|$ is given by

$$
\begin{aligned}
\left\|e^{\check{A} t}\right\| & =\left|e^{(\cos \theta-1+j \sin (\theta)) t}\right|\left\|\left[\begin{array}{ll}
1 & t \\
0 & 1
\end{array}\right]\right\| \\
& =\left|e^{(\cos \theta-1) t}\right| \sqrt{\frac{2+t^{2}+\sqrt{t^{4}+4 t^{2}}}{2}}
\end{aligned}
$$

which tends to infinity as $t \rightarrow \infty$.

A Lyapunov-type condition follows from [5, Theorem 5.1.3] 
Theorem 3.7: An operator $\check{A} \in \mathbf{L}_{\infty}\left(\partial \mathbb{D} ; \mathbb{C}^{n \times n}\right)$ generates an exponentially stable semigroup if and only if there exists a positive operator $\check{P} \in \mathbf{L}_{\infty}\left(\partial \mathbb{D} ; \mathbb{C}^{n \times n}\right)$ such that

$$
\check{A}^{*} \check{P}+\check{P}^{*} \check{A}=-I
$$

If $A \in \ell_{1}\left(\mathbb{C}^{n \times n}\right)$, then $P \in \ell_{1}\left(\mathbb{C}^{n \times n}\right)$ and so $\check{P}\left(e^{j \theta}\right)$ will be continuous in $\theta$ on $[0,2 \pi]$.

Proof We only need to prove the continuity. The solution to the isometrically isomorphic Lyapunov equation $A^{*} P+$ $P^{*} A=-I$ is

$$
P=\int_{0}^{\infty} e^{A^{*} t} e^{A t} \mathrm{~d} t
$$

Now $\ell_{1}\left(\mathbb{C}^{n \times n}\right)$ is a Banach algebra and so closed under limits. Hence $e^{A t} \in \ell_{1}\left(\mathbb{C}^{n \times n}\right)$ and so is the integrand. Now $e^{A t}$ is exponentially stable and so for some $M, \alpha>0$

$$
\left\|e^{A^{*} t} e^{A t}\right\| \leq M^{2} e^{-2 \alpha t} .
$$

By the Lebesgue lemma we conclude that $P \in \ell_{1}\left(\mathbb{C}^{n \times n}\right)$ which implies that $\check{P}\left(e^{j \theta}\right)$ is continuous.

For Example 3.3 the Lyapunov equation has the solution

$$
\check{P}\left(e^{j \theta}\right)=-\frac{1}{2[(b+c) \cos \theta+a]},
$$

which shows that $e^{\check{A} t}$ will generate an exponentially stable semigroup if and only if $-a>|b+c|$.

The Lyapunov equation associated to the system considered in Example 3.5 does not have a positive solution which confirms our earlier conclusion that $e^{A t}$ does not generate an exponentially stable semigroup.

\section{Controllability AND OBSERVABility}

Since $\Sigma(A, B, C, D)$ is isometrically isomorphic to the state linear system $\Sigma(\breve{A}, \breve{B}, \breve{C}, \breve{D})$, their approximate controllability and observability properties are identical. It is more convenient to analyze the Fourier transformed system to deduce the properties of $\Sigma(A, B, C, D)$. The necessary and sufficient conditions for approximate controllability from [5, Definition 4.1.17] applied to $\Sigma(\check{A}, \breve{B}, \check{C}, \check{D})$ yield

$$
\breve{B}^{*} e^{\check{A}^{*}}{ }_{\breve{z}}=0 \text { for } t \geq 0 \Longrightarrow \check{z}=0,
$$

and for the dual concept of approximate observability they yield

$$
\check{C} e^{\check{A} t} \check{z}=0 \text { for } t \geq 0 \Longrightarrow \check{z}=0 .
$$

Since $\check{A}, \breve{B}, \check{C}$ are bounded operators, we obtain the following necessary and sufficient condition for approximate observability from the dual of [4, Theorem 3.16]

$$
\operatorname{ker}\left[\begin{array}{c}
\check{C} \\
\check{C} \check{A} \\
\ldots \\
\check{C} \check{A}^{r} \\
\cdots
\end{array}\right]=\{0\} .
$$

Moreover, since $\check{A}$ and $\check{C}$ have matrix values, this condition reduces to

$$
\operatorname{ker}\left[\begin{array}{c}
\check{C}\left(e^{j \theta}\right) \\
\check{C}\left(e^{j \theta}\right) \check{A}\left(e^{j \theta}\right) \\
\check{C}\left(e^{j \theta}\right) \ddot{\check{A}}\left(e^{j \theta}\right)^{n-1}
\end{array}\right]=\{0\}
$$

for almost all $\theta \in[0,2 \pi]$.

Similarly, we obtain the following necessary and sufficient condition for approximate controllability

$$
\operatorname{rank}\left[\check{B}\left(e^{j \theta}\right): \check{A}\left(e^{j \theta}\right) \check{B}\left(e^{j \theta}\right): \ldots \check{A}\left(e^{j \theta}\right)^{n-1} \check{B}\left(e^{j \theta}\right)\right]=n
$$

for almost all $\theta \in[0,2 \pi]$.

As in the finite-dimensional case, this leads to the following Hautus test.

Lemma 4.1: $\Sigma(\check{A}, \check{B}, \check{C}, \check{D})$ is approximately controllable if and only if

$$
\operatorname{rank}\left[\left(\lambda I-\check{A}\left(e^{j \theta}\right)\right): \check{B}\left(e^{j \theta}\right)\right]=n
$$

for almost all $\theta \in[0,2 \pi]$ and for all $\lambda \in \mathbb{C}$.

$\Sigma(\breve{A}, \breve{B}, \breve{C}, \breve{D})$ is approximately observable if and only if

$$
\operatorname{rank}\left[\begin{array}{c}
\lambda I-\check{A}\left(e^{j \theta}\right) \\
\check{C}\left(e^{j \theta}\right)
\end{array}\right]=n
$$

for almost all $\theta \in[0,2 \pi]$ and for all $\lambda \in \mathbb{C}$.

One might expect that, in the case that $\check{A}\left(e^{j \theta}\right), \check{B}\left(e^{j \theta}\right), \check{C}\left(e^{j \theta}\right)$ are continuous in $\theta$ on $[0,2 \pi]$, necessary and sufficient conditions for approximate observability and controllability should be that (9) and (10) hold for all $\theta \in[0,2 \pi]$. The following example shows that there are approximately controllable systems with $\check{A}\left(e^{j \theta}\right), \breve{B}\left(e^{j \theta}\right), \check{C}\left(e^{j \theta}\right)$ continuous in $\theta$ on $[0,2 \pi]$ for which the rank condition does not hold for all $\theta \in[0,2 \pi]$.

Example 4.2: Consider the system (3) with

$$
A_{0}=\left[\begin{array}{ll}
0 & 1 \\
0 & 0
\end{array}\right], B_{0}=\left[\begin{array}{l}
0 \\
1
\end{array}\right], B_{1}=\left[\begin{array}{c}
0 \\
-1
\end{array}\right]
$$

and all other $A_{l}, B_{l}$ zero. To examine the approximate controllability of this system we examine the Fourier transformed system which has the operators

$$
\check{A}=\left[\begin{array}{ll}
0 & 1 \\
0 & 0
\end{array}\right], \quad \check{B}\left(e^{j \theta}\right)=\left[\begin{array}{c}
0 \\
1-e^{-j \theta}
\end{array}\right] .
$$

We have

$$
\begin{aligned}
\check{B}\left(e^{j \theta}\right)^{*} e^{\check{A}\left(e^{j \theta}\right)^{*} t}\left[\begin{array}{l}
\xi \\
\rho
\end{array}\right] & =\left[01-e^{-j \theta}\right]\left[\begin{array}{ll}
1 & 0 \\
t & 1
\end{array}\right]\left[\begin{array}{l}
\xi \\
\rho
\end{array}\right] \\
& =\left(1-e^{-j \theta}\right)(t \xi+\rho) .
\end{aligned}
$$

If this equals zero almost everywhere in $[0,2 \pi]$ for $t \geq 0$, we must have $\xi=0=\rho$. Consequently it is approximately controllable, even though it does not satisfy the rank condition (10) in $\theta=0$.

It turns out that, in the case that $\check{A}\left(e^{j \theta}\right), \check{B}\left(e^{j \theta}\right), \check{C}\left(e^{j \theta}\right)$ are continuous in $\theta$ on $[0,2 \pi]$, if (9) and (10) hold for all $\theta \in$ $[0,2 \pi]$, then the system is exactly controllable. 
We recall that $\Sigma(\check{A}, \check{B}, \check{C} \check{D})$ is exactly controllable on $[0, T]$ if and only if there exists a positive $\beta$ such that

$$
\int_{0}^{T}\left\|\check{B}^{*} e^{\check{A}^{*} t} \check{z}_{\mathbf{L}_{2}\left(\partial \mathbb{D} ; \mathbb{C}^{m}\right)}^{2} \mathrm{~d} t \geq \beta\right\|\left\|_{\tilde{z}}\right\|_{\mathbf{L}_{2}\left(\partial \mathbb{D} ; \mathbb{C}^{n}\right)}^{2},
$$

and the dual concept for exactly observable on $[0, T]$ if and only if there exists a positive $\gamma$ such that

$$
\int_{0}^{T}\left\|\check{C} e^{\check{A} t} \check{z}\right\|_{\mathbf{L}_{2}\left(\partial \mathbb{D} ; \mathbb{C}^{p}\right)}^{2} \mathrm{~d} t \geq \gamma\|\check{z}\|_{\mathbf{L}_{2}\left(\partial \mathbb{D} ; \mathbb{C}^{n}\right)}^{2} .
$$

Lemma 4.3: Suppose that $\check{A}\left(e^{j \theta}\right), \check{B}\left(e^{j \theta}\right)$ and $\check{C}\left(e^{j \theta}\right)$ are continuous in $\theta$ on $[0,2 \pi]$. Then $\Sigma(\breve{A}, \breve{B}, \check{C}, \check{D})$ is exactly controllable if and only if

$$
\operatorname{rank}\left[\left(\lambda I-\check{A}\left(e^{j \theta}\right)\right): \check{B}\left(e^{j \theta}\right)\right]=n
$$

for all $\theta \in[0,2 \pi]$ and for all $\lambda \in \mathbb{C}$.

$\Sigma(\breve{A}, \breve{B}, \breve{D})$ is exactly observable if and only if

$$
\operatorname{rank}\left[\begin{array}{c}
\lambda I-\check{A}\left(e^{j \theta}\right) \\
\check{C}\left(e^{j \theta}\right)
\end{array}\right]=n
$$

for all $\theta \in[0,2 \pi]$ and for all $\lambda \in \mathbb{C}$.

Proof. Since both concepts are dual, it suffices to prove the exact observability result.

Necessity: It can be proved by contradiction.

Sufficiency: From [5, Lemma 4.1.6] we can assume that the system is exponentially stable. Consequently, one can use the sufficient condition for exact observability from [20, Theorem 1.7] to establish this implication.

\section{EXPONENTIAL STABILIZABILITY AND DETECTABILITY}

We recall that $\Sigma(A, B, C, D)$ is exponentially stabilizable if there exists a $F \in \mathcal{L}(Z, U)$ such that $A+B F$ is exponentially stable and it is exponential detectable if there exists a $L \in \mathcal{L}(Y, Z)$ such that $A+L C$ is exponentially stable. We derive simple conditions for exponential stabilizability and exponential detectability by analyzing its Fourier transformed system.

Lemma 5.1: Suppose that $\check{A}, \breve{B}, \check{C}$ are continuous in $\theta$ on $[0,2 \pi]$.

$\Sigma(\check{A}, \check{B}, \check{C}, 0)$ is exponentially stabilizable if and only if $\left(\check{A}\left(e^{j \theta}\right), \breve{B}\left(e^{j \theta}\right)\right)$ is exponentially stabilizable for each $\theta \in$ $[0,2 \pi]$, i.e.,

$$
\operatorname{rank}\left[\left(\lambda I-\check{A}\left(e^{j \theta}\right)\right): \check{B}\left(e^{j \theta}\right)\right]=n
$$

for all $\theta \in[0,2 \pi]$ and for all $\lambda \in \overline{\mathbb{C}}_{0}^{+}$.

$\Sigma(\check{A}, \breve{B}, \breve{C}, 0)$ is exponentially detectable if and only if $\left(\check{A}\left(e^{j \theta}\right), \check{C}\left(e^{j \theta}\right)\right)$ is exponentially detectable for each $\theta \in$ $[0,2 \pi]$, i.e.,

$$
\operatorname{rank}\left[\begin{array}{c}
\lambda I-\check{A}\left(e^{j \theta}\right) \\
\check{C}\left(e^{j \theta}\right)
\end{array}\right]=n
$$

for all $\theta \in[0,2 \pi]$ and for all $\lambda \in \overline{\mathbb{C}}_{0}^{+}$,

Proof It suffices to prove the exponential stabilizability condition.
Sufficiency: One can use the unique non-negative stabilizing solution to the Riccati equation

$$
\begin{array}{r}
\check{A}\left(e^{j \theta}\right)^{*} \check{Q}\left(e^{j \theta}\right)+\check{Q}\left(e^{j \theta}\right) \check{A}\left(e^{j \theta}\right) \\
-\check{Q}\left(e^{j \theta}\right) \check{B}\left(e^{j \theta}\right) \check{B}\left(e^{j \theta}\right)^{*} \check{Q}\left(e^{j \theta}\right)+I=0 .
\end{array}
$$

and [13, Theorem 11.2.1]. It can be shown that $\check{A}-\check{B} \breve{B}^{*} \check{Q}$ generates an exponentially stable semigroup.

Necessity: It can be proved by contradiction.

Corollary 5.2: If $\check{A}, \check{B}, \check{C}$ are summable, then there exists a summable $\breve{F}$ such that $\breve{A}+\breve{B} \breve{F}$ generates an exponentially stable semigroup if and only if (13) holds. Moreoever, $\Sigma(\breve{A}, \breve{B}, \breve{C},-)$ is exponentially stabilizable (detectable) with respect to the spaces if and only if (13) ((14)) holds.

Proof. Since the summable norm is strictly larger than the infinity norm, we need only prove sufficiency.

First we show the existence of a summable $\check{F}$. From Lemma 5.1 we have a stabilizing $\check{F}=-\breve{B}^{*} \check{Q}$ so that there exist positive constants $M, \alpha$ such that

$$
\left\|e^{(\check{A}+\breve{B} \breve{F}) t}\right\|_{\infty} \leq M e^{-\alpha t} .
$$

Since $\check{F}\left(e^{j \theta}\right)$ is continuous and periodic on $[0,2 \pi]$, it is approximable by

$$
\check{F}^{N}=\sum_{l=-N}^{N} F_{l} e^{-j l \theta}
$$

in the sup norm ([21, Proposition 1, p.113]). Choose $N$ sufficiently large so that

$$
\left\|\check{F}-\check{F}^{N}\right\|_{\infty}<\varepsilon=\frac{\alpha}{2 M\|\check{B}\|} .
$$

Then using the perturbation result from [5, Theorem 3.2.1] we have

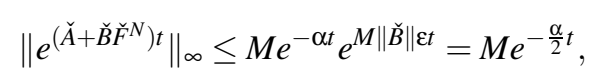

which shows exponential stability in the $\mathbf{L}_{\infty}$-norm. Hence the spectrum lies in the open left half-plane. From Lemma, $\check{A}+\breve{B} \breve{F}$ generates and exponentially stable semigroup on both spaces.

We remark that in Lemma 5.1 it is essential that $\check{A}\left(e^{j \theta}\right)$, $\check{B}\left(e^{j \theta}\right)$ and $\check{C}\left(e^{j \theta}\right)$ be continuous in $\theta$. Unlike the approximate controllability condition, the rank condition for exponential stabilizability should hold for all $\theta \in[0,2 \pi]$ as the following example illustrates.

Example 5.3: Consider the system from Example 4.2.

$$
\check{A}\left(e^{j \theta}\right)=\left[\begin{array}{ll}
0 & 1 \\
0 & 0
\end{array}\right], \quad \check{B}\left(e^{j \theta}\right)=\left[\begin{array}{c}
0 \\
1-e^{-j \theta}
\end{array}\right] .
$$

The Riccati equation has the unique positive solution

$$
\check{Q}\left(e^{j \theta}\right)=\left[\begin{array}{cc}
\sqrt{1+1 / \sin \theta / 2} & 1 / 2 \sin \theta / 2 \\
1 / 2 \sin \theta / 2 & \frac{\sqrt{1+\sin \theta / 2}}{2 \sin ^{3} \theta / 2}
\end{array}\right]
$$

for each $\theta \in[0,2 \pi]$, but $\check{Q}\left(e^{j \theta}\right)$ is not bounded on $[0,2 \pi]$. So the operator Riccati equation

$$
\check{A}^{*} \check{Q}+\check{Q} \check{A}-\check{Q} \check{B} \check{B}^{*} \check{Q}+I=0
$$


does not have a nonnegative self-adjoint solution. From Theorem 6.2.4 in [5] we conclude that $\Sigma(\check{A}, \check{B}, \check{C}, \check{D})$ is not exponentially stabilizable. This checks with the observation that the condition (13) fails for $\theta=0$.

So for this class of systems approximate controllability does not imply exponential stabilizability.

\section{CONCLUSIONS AND FUTURE WORK}

We have considered a class of infinite-dimensional systems which have a spatially invariant dynamics. The motivation for studying this special class of system stems from the interest shown in the literature for controlling infinite platoons of vehicles over the years. Testable criteria for various system theoretic properties have been derived and illustrated with simple examples. Further research will focus on the relationship between the LQR problem for very large, but finite, platoons of vehicles and the LQR problem for an infinite platoon.

\section{REFERENCES}

[1] W. Arendt and C.J.K. Batty. Tauberian theorems and stability of one-parameter semigroups Transactions of American Mathematical Society, 306:837-841, 1988.

[2] B. Bamieh and F. Paganini and M.A. Dahleh. Distributed control of spatially invariant systems. IEEE Trans. Automatic Control 47:10911107, 2002.

[3] K.C. Chu. Optimal decentralized regulation for a string of coupled systems. IEEE Trans. Automatic Control 19:243-246, 1974.

[4] R.F. Curtain and A.J. Pritchard. Infinite-Dimensional Linear Systems Theory, vol. 8 in Lecture Notes in Control and Information Sciences Springer-Verlag, Berlin, 1978.

[5] R.F. Curtain and H.J. Zwart. An Introduction to Infinite-Dimensional Linear Systems Theory. Springer-Verlag, New York, 1995.

[6] M L.El-Sayed and P.S. Krishnaprasad. Homogeneous interconnected systems: an example, IEEE Trans, A-C 26: PP894-901, 1981.

[7] P.L. Falb and M.I. Freedman. A Generalized transform theory for causal operators. SIAM. J. Control, 7:452-471, 1969.

[8] M.I. Freedman and P.L. Falb and G. Zames. Hilbert space stability theory. SIAM. J. Control, 7:479-495, 1969.

[9] I. Gohberg and S. Goldberg and M.A. Kaashoek Classes of Linear Operators, Vol. II, volume 63 of Operator Theory, Advances and Applications Birkhäuser Verlag, Basel, 1993.

[10] W.L. Green and E.W. Kamen. Stabilizability of linear systems over a commutative normed linear algebra with applications to spatiallydistributed and parameter-dependent systems. SIAM Journal on Control and Optimization, 23:1-18, 1985.

[11] E.W. Kamen and W.L. Green. Asymptotic stability of linear difference equations defined over a commutative Banach algebra. J. Math. Anal. Appl., 75:584-601, 1980.

[12] M.R. Jovanović and B. Bamieh. On the ill-posedness of certain vehicular platoon control problems. IEEE Trans. Automatic Control 50:1307-1321, 2005.

[13] P. Lancaster and L. Rodman. Algebraic Riccati Equations. Oxford Science Publications, Clarendon Press, Oxford ,UK, 1995.

[14] W.S Levine and A. Athans. On the optimal error egulation of a string of moving vehicles. IEEE Trans. Automatic Control 11:355-361, 1966.

[15] L. Loomis An Introduction to Abstract Harmonic Analysis Van Nostrand, Princeton, 1953.

[16] S.M. Melzer and B.C. Kuo. Optimal regulation of systems described by a countably infinite number of objects. Automatica 11:359-366, 1971.

[17] S.M. Melzer and B.C. Kuo. A closed form solution for the optimal error regulation of a string of moving vehicles. IEEE Trans. Automatic Control 16:50-52, 1971.

[18] K.A. Morris. Convergence of controllers designed using state-space methods. IEEE Trans. Autom. Control;; 39:2100-2104, 1994.

[19] W. Rudin. Fourier Analysis on Groups Interscience, New York, 1962.

[20] D.L. Russell and G. Weiss. A general necessary condition for exact observability. SIAM J. Control Optimization 32:1-23, 1994.
[21] R.M. Young. An Introduction to Nonharmonic Fourier Series Academic Press, New York, 1980.

\section{APPENDIX: FREQUENCY-DOMAIN SPACES}

In this section we recall the definitions and introduce the notations for various frequency domain spaces with respect to the unit disc.

Definition 7.1: Denote by $\mathbb{D}$ the unit disc $\{z \in \mathbb{C}|| z \mid<1\}$ and by $\partial \mathbb{D}$ its boundary, the unit circle $\{z \in \mathbb{C}|| z \mid=1\}$. We define the following frequency-domain spaces:

$$
\begin{aligned}
L_{2}\left(\partial \mathbb{D} ; \mathbb{C}^{m}\right)= & \left\{f: \partial \mathbb{D} \rightarrow \mathbb{C}^{m} \mid f\right. \text { is measurable and } \\
& \left.\|f\|_{2}=\left(\frac{1}{2 \pi} \int_{0}^{2 \pi}\left|f\left(e^{j \theta}\right)\right|^{2} d \theta\right)^{\frac{1}{2}}<\infty\right\} \\
L_{\infty}\left(\partial \mathbb{D} ; \mathbb{C}^{k \times m}\right)= & \left\{F: \partial \mathbb{D} \rightarrow \mathbb{C}^{k \times m} \mid F\right. \text { is measurable } \\
\text { and } & \left.\|F\|_{\infty}=\underset{0<\theta \leq 2 \pi}{\operatorname{ess} \sup }\left\|F\left(e^{j \theta}\right)\right\|<\infty\right\}
\end{aligned}
$$

$L_{2}\left(\partial \mathbb{D} ; \mathbb{C}^{m}\right)$ is a Hilbert space under the inner product

$$
\left\langle f_{1}, f_{2}\right\rangle_{2}=\frac{1}{2 \pi} \int_{0}^{2 \pi}\left\langle f_{1}\left(e^{j \theta}\right), f_{2}\left(e^{j \theta}\right)\right\rangle_{\mathbb{C}^{m}} d \theta .
$$

$L_{\infty}\left(\partial \mathbb{D} ; \mathbb{C}^{k \times m}\right)$ is a Banach space under the $\|\cdot\|_{\infty}$-norm. Its elements induce a bounded operator from $L_{2}\left(\partial \mathbb{D} ; \mathbb{C}^{m}\right)$ to $L_{2}\left(\partial \mathbb{D} ; \mathbb{C}^{k}\right)$.

Property 7.2: If $F \in L_{\infty}\left(\partial \mathbb{D} ; \mathbb{C}^{k \times m}\right)$ and $u \in L_{2}\left(\partial \mathbb{D} ; \mathbb{C}^{m}\right)$, then $F u \in L_{2}\left(\partial \mathbb{D} ; \mathbb{C}^{k}\right)$. The multiplication map $\Lambda_{F}: u \mapsto$ $F u$ defines a bounded linear operator from $L_{2}\left(\partial \mathbb{D} ; \mathbb{C}^{m}\right) \rightarrow$ $L_{2}\left(\partial \mathbb{D} ; \mathbb{C}^{k}\right)$ (often called a Laurent operator) and

$$
\left\|\Lambda_{F}\right\|=\sup _{u \neq 0} \frac{\left\|\Lambda_{F} u\right\|_{L_{2}\left(\partial \mathbb{D} ; \mathbb{C}^{k}\right)}}{\|u\|_{L_{2}\left(\partial \mathbb{D} ; \mathbb{C}^{m}\right)}}=\|F\|_{\infty} .
$$

If $k=m$, we obtain the Banach algebra $L_{\infty}\left(\partial \mathbb{D} ; \mathbb{C}^{k \times k}\right)$. We quote the following result from Gohberg et al $[9$, Theorem 2.4, p.569] on the existence of an inverse.

Lemma 7.3: $L_{\infty}\left(\partial \mathbb{D} ; \mathbb{C}^{k \times k}\right)$ is a Banach algebra and $F \in$ $L_{\infty}\left(\partial \mathbb{D} ; \mathbb{C}^{k \times k}\right)$ is boundedly invertible if and only if $\exists$ a $\gamma>0$ such that $\left\{\theta|| \operatorname{det}\left(F\left(e^{j \theta}\right) \mid<\gamma\right\}\right.$ has measure zero. If $F$ is continous, then $F \in L_{\infty}\left(\partial \mathbb{D} ; \mathbb{C}^{k \times k}\right)$ is boundedly invertible if and only if $\operatorname{det} F\left(e^{j \theta}\right) \neq 0$ for all $\theta \in \partial \mathbb{D}$.

Elements of $\mathbf{L}_{2}(\partial \mathbb{D})$ arise naturally as Fourier transforms of elements in $\ell_{2}\left(\mathbb{C}^{m}\right)=\left\{z=\left(z_{r}\right)_{n=-\infty}^{\infty}, z_{r} \in\right.$ $\left.\mathbb{C}^{m} \mid \sum_{r=-\infty}^{\infty}\left\|z_{r}\right\|_{\mathbb{C}^{m}}^{2}<\infty\right\}$.

Definition 7.4: The Fourier transform of an element of $z \in \ell_{2}\left(\mathbb{C}^{m}\right)$ is defined by

$$
\check{z}\left(e^{j \theta}\right)=\sum_{r=-\infty}^{\infty} z_{r} e^{-j r \theta}, \quad \theta \in \mathbb{D},
$$

which is precisely the Fourier series representation of an element $\check{z} \in \mathbf{L}_{2}\left(\partial \mathbb{D} ; \mathbb{C}^{m}\right)$ with the Fourier coefficients $z_{r}=$ $\frac{1}{2 \pi} \int_{0}^{2 \pi} \check{z}\left(e^{j \theta}\right) e^{j r \theta} \mathrm{d} \theta$.

Note that an element $\check{z} \in \mathbf{L}_{2}\left(\partial \mathbb{D} ; \mathbb{C}^{m}\right)$ has the inverse Fourier transform $z=\left(z_{r}\right)_{r=-\infty}^{\infty}, z_{r} \in \mathbb{C}^{m}$, where $z_{r}$ are the Fourier coefficients of $\check{z}$.

Moreover, ${ }^{\vee}: \ell_{2}\left(\mathbb{C}^{m}\right) \rightarrow \mathbf{L}_{2}\left(\partial \mathbb{D} ; \mathbb{C}^{m}\right)$ is an isometric isometry with $\|\check{z}\|_{\mathbf{L}_{2}\left(\partial \mathbb{D} ; \mathbb{C}^{m}\right)}=\|z\|_{\ell_{2}\left(\mathbb{C}^{m}\right)}$. 